\title{
ESTADO NUTRIGIONAL, ANEMIA E PARASITOSES INTESTINAIS EM GESTANTES DE UM MUNICIPIO DO CURIMATAÚ PARAIBANO
}

Nutricional status, anemia and enteroparasites prevalence in pregnant of a city of Curimataú - Paraiba

Arthur Silva Bezerra, ${ }^{1}$ Vanille Valério Barbosa Pessoa Cardoso², Vanessa Santos de Arruda Barbosa ${ }^{3}$

\section{RESUMO}

O presente estudo objetivou conhecer o perfil socioeconômico, nutricional, hematológico e determinar a prevalência de infecção por enteroparasitos em gestantes do município de Cuité - PB. A coleta de dados realizou-se na Estratégia Saúde da Família entre os meses de maio a julho de 2014. Os dados foram obtidos por meio de um questionário semiestruturado e entrevistas livres. Foram feitos exames parasitológicos de fezes pelo método de sedimentação espontânea e coletados resultados dos exames hematológicos e de características obstétricas do cartão da gestante. Para avaliação nutricional, foi utilizada balança antropométrica com estadiômetro para aferição de peso e altura. Utilizou-se o teste Qui-quadrado $(\mathrm{p}<0,05)$ para se avaliar associação entre variáveis e os dados foram processados no programa estatístico SPSS. Das 45 gestantes que participaram da pesquisa, a maioria relatou possuir apenas o ensino médio e renda mensal de até um salário mínimo. A maior parte apresentou estado nutricional eutrófico $(48,5 \%)$. A prevalência de enteroparasitoses encontrada foi de $48 \%$, sendo os enteroparasitos mais frequentes: Entamoeba coli e E.histolytica/E.dispar. A utilização de água de procedência inadequada para beber mostrou-se associada à infecção por parasitas intestinais $(\mathrm{p}=0,027)$. Observou-se uma prevalência baixa de anemia. Os resultados apontam para a existência de uma população exposta a riscos de infecção por enteroparasitos.

PALAVRAS-CHAVE: Enteropatias Parasitárias; Cuidado Pré-Natal; Saúde Pública.

\begin{abstract}
This study's purpose was to ascertain the socioeconomic, nutritional, and hematological profile and determine the prevalence of enteroparasites in pregnant women of Cuité city, Paraíba. Data was collected in the Family Health Program between May and July of 2014. Data were obtained through a semi-structured questionnaire and free interviews. Parasitological stool tests were performed using the spontaneous sedimentation method. Results of hematology exams and obstetrical characteristics from the women's records were collected. For nutritional assessment an anthropometric scale with stadiometer was used for measuring weight and height. The chi-square test $(\mathrm{p}<0.05)$ was used to evaluate the association between variables. Data were processed using the SPSS statistical program. Most of the 45 pregnant women that participated in the research reported having only a high school education and having a family income no greater than minimum wage in Brazil. A large number of the pregnant women presented a eutrophic nutritional status $(48.5 \%)$. The prevalence of enteroparasitoses found was $48 \%$, the most common enteroparasites being: Entamoeba coli and E.bistolytica/E.dispar. The use of water of inadequate origin for drinking was associated with the infection by intestinal parasites $(p=0.027)$. A low prevalence of anemia was observed among the pregnant women. The results point to the existence of a population exposed to risk of infection by enteroparasites.
\end{abstract}

KEYWORDS: Intestinal Diseases; Parasitic; Prenatal Care; Public Health.

\footnotetext{
1 Graduado em Ciências Farmacêuticas, Unidade Acadêmica da Educação, Centro de Educação e Saúde (CES), Universidade Federal de Campina Grande (UFCG).

2 Msc. Professora Assistente, Núcleo de Pesquisas e Estudos em Nutrição e Saúde Coletiva, Unidade Acadêmica da Sáude (UAS), CES, UFCG

3 Dra. Professora Adjunto, Laboratório de Parasitologia, UAS, CES, UFCG.
} 


\section{INTRODUÇÃO}

Apesar dos avanços tanto na área farmacológica quanto nas medidas de saneamento básico e educação sanitária, as enteroparasitoses ainda são um sério problema de saúde pública. As estimativas são de que mais de um bilhão de pessoas em todo o mundo alberguem pelo menos uma espécie de parasita intestinal, sendo amebíase, ascaridíase, tricuríase e a ancilostomíase as enteroparasitoses com maior prevalência mundial. ${ }^{1}$

No Brasil, as parasitoses intestinais apresentam uma ampla distribuição geográfica, ocorrendo nas diversas regiões. ${ }^{2,3,4}$ Ausência de ou insuficiente condição mínima de saneamento básico e inadequadas práticas de higiene pessoal e doméstica, contato com animais domésticos e outros fatores de ordem econômica, social e cultural são altamente relevantes para a transmissão e ampla disseminação dos parasitos. ${ }^{5}$

As enteroparasitoses podem afetar o equilíbrio nutricional, pois interferem na absorção de nutrientes e induzem sangramento intestinal e ainda podem causar complicações significativas, como obstrução intestinal. ${ }^{6}$ Os estágios transmissíveis dos parasitos (ovos, larvas, cistos e oocistos) podem ser encontrados no ambiente, quer no solo, água ou alimentos, como consequência da contaminação direta ou indireta com fezes humanas ou de animal. As helmintíases mais comuns são: ancilostomíase, ascaridíase, enterobiose, estrongiloidíase, himenolepíase, teníase e tricuríase. Já as protozooses intestinais mais encontradas são: giardíase e amebíase. ${ }^{7}$

A associação entre gravidez e parasitoses deve ser investigada durante a rotina do pré-natal, ${ }^{8}$ sendo recomendado o exame coproparasitológico e o tratamento da parasitose durante a gestação. ${ }^{9}$ A parasitose intestinal na gestação pode representar fator de risco não declarado de anemia e crescimento intrauterino restrito (CIUR). ${ }^{10}$

Clinicamente, as enteroparasitoses na gestação podem provocar efeitos patológicos diretos e cursar com sintomas digestivos leves, como dor abdominal, náuseas, diarreia ou constipação intestinal, digestão difícil e flatulência, podendo tais sintomas, em algumas ocasiões, ser confundidos com manifestações próprias do início da gravidez. ${ }^{11}$ Há relato na literatura sobre aumento significativo na incidência de retardamento de desenvolvimento intrauterino em mulheres infectadas com Ascaris lumbricoides e Trichuris trichiura. $^{5,12}$

O diagnóstico de uma enteroparasitose durante a gestação objetiva a busca do bem-estar físico da gestante e de seu feto, por meio de tratamento antiparasitário específico e orientações profiláticas, evitando que passem simplesmente de gestantes parasitadas a mães parasitadas e que, consequentemente, transmitam as formas infectantes para a criança. Entretanto um diagnóstico positivo para parasitos intestinais, muitas vezes, é ignorado durante o pré-natal. ${ }^{11,13}$

Infecções parasitárias também podem exercer importante influência sobre o estado nutricional e ser fator de risco para desenvolvimento de anemia em gestantes. ${ }^{14} \mathrm{O}$ Ministério da Saúde também ressalta a importância de se associarem parasitoses ao agravamento da anemia fisiológica ou da ferropriva, tão comuns na gravidez. ${ }^{8}$

Além da presença de parasitos intestinais, o prognóstico da gestação também é influenciado pelo estado nutricional materno antes e durante a gravidez. A inadequação do estado nutricional materno tem grande impacto sobre o crescimento e desenvolvimento do recém-nascido, pois o período gestacional é uma fase na qual as necessidades nutricionais são elevadas, decorrentes dos ajustes fisiológicos da gestante e das demandas de nutrientes para o crescimento fetal. ${ }^{15,16}$

Diante desse contexto, o estudo teve como objetivo conhecer o perfil socioeconômico, hematológico e a prevalência dos helmintos e protozoários intestinais, por meio de exames parasitológicos de fezes, em gestantes acompanhadas pela Estratégia de Saúde da Família do município de Cuité - PB.

\section{MATERIAIS E MÉTODOS}

Trata-se de um estudo epidemiológico do tipo transversal, baseado em um inquérito para avaliar a prevalência de parasitoses intestinais de anemia e as condições socioeconômicas e sanitárias de gestantes do município de Cuité no período de maio a julho de 2014. Este se situa na mesorregião do Agreste Paraibano e na microrregião do Curimataú Ocidental, com população estimada em 19.978 habitantes em 2010, segundo o Instituto Brasileiro de Geografia e Estatística (IBGE). ${ }^{17} \mathrm{O}$ município contava, no período da pesquisa, com oito Equipes de Saúde da Família, cinco na zona urbana e três na zona rural. O estudo teve como alvo 66 gestantes cadastradas e acompanhadas pelas ESFs e residentes na zona urbana de Cuité no período de maio a julho de 2014. A amostragem foi do tipo não probabilística de conveniência ${ }^{18} \mathrm{e}$ se estabeleceram como critérios de inclusão: a) gestantes cadastradas e acompanhadas pela Estratégia de Saúde da Família da zona urbana do município de Cuité; b) gestantes que assinaram o Termo de Consentimento Livre e Esclarecido (TCLE) ou que tiveram o TCLE assinado por responsável legal por serem menores de idade.

A coleta de dados foi realizada em cada uma das cinco Equipes de Saúde da Família da zona urbana do municí- 
pio. Como instrumentos de coleta de dados, utilizaram-se questionário semiestruturado e entrevistas livres para se avaliarem as condições sociais e higiênico-sanitárias. Foram coletados do cartão da gestante resultados dos exames hematológicos e de características obstétricas realizados ao longo do pré-natal. A avaliação nutricional das gestantes ocorreu no espaço físico da Unidade de Saúde da Família na qual a gestante estava cadastrada. Foi utilizada balança antropométrica com estadiômetro do próprio serviço para aferição de peso e altura.

Para a coleta das amostras fecais, foram distribuídos coletores universais de plástico apropriados e as gestantes foram orientadas quanto ao procedimento padrão de coleta de uma única amostra. Os coletores com as amostras de fezes que foram devolvidas pelas voluntárias foram encaminhados ao Laboratório de Parasitologia do CES/ UFCG. Foram realizados exames coproparasitológicos pelo método de sedimentação espontânea. Todas as lâminas foram analisadas em triplicata, utilizando-se microscópico óptico com objetivas de 10x e 40x. ${ }^{19}$

Considerou-se anêmica a gestante com hemoglobina inferior a $11,0 \mathrm{~g} / \mathrm{dL}$, de acordo com diretrizes do Ministério da Saúde. ${ }^{8}$ Consideraram-se como de Baixa Escolaridade as gestantes com Nenhum Estudo, Alfabetizadas e com Ensino Fundamental. Para Alta Escolaridade, consideraram-se as gestantes com Ensino Médio e Ensino Superior. Consideraram-se como tendo Esgotamento Sanitário Adequado as gestantes com residências em que tal esgotamento sanitário fosse Rede Pública ou Fossa Séptica. Na Condição de Risco, consideraram-se as gestantes com residências em que o esgotamento sanitário fosse Fossa Negra, Vala ou com Ausência de Esgotamento Sanitário. Quanto à água consumida, consideraram-se adequados os tipos: água fervida, água mineral ou água filtrada; e inadequados: água coada e água de procedência desconhecida.

Todos os dados foram armazenados inicialmente no programa Microsoft Access e, posteriormente, a análise estatística foi realizada utilizando-se o software Statistical Package for Social Sciences (SPSS), versão 13.0. Associações estatísticas entre as variáveis foram avaliadas por meio do teste qui-quadrado e valores de $\mathrm{p}<0,05$ foram considerados estatisticamente significantes. $\mathrm{Na}$ estatística descritiva, utilizaram-se o número absoluto e a frequência relativa das variáveis de maior relevância para a construção de tabelas.

O trabalho foi aprovado pelo Comitê de Ética e Pesquisa para Seres Humanos do Hospital Universitário Alcides Carneiro (Parecer no 575.704).

\section{RESULTADOS}

Dentre as 66 gestantes em atendimento pré-natal na zona urbana de Cuité, durante o período do estudo, 45 aceitaram participar da pesquisa. Com relação ao perfil socioeconômico da população estudada, verificou-se que a ocupação mais citada pelas gestantes foi a de agricultora $(28,9 \%)$ e, em seguida, a de doméstica (26,7\%). A Tabela 1 mostra a distribuição quanto à faixa etária e estado civil. Os dados quanto à escolaridade mostram que 53,3\% possuíam o ensino médio, $26,7 \%$ possuíam o ensino fundamental e $17,8 \%$, o ensino superior, e apenas uma não possuía nenhum estudo.

Tabela 1 - Percentual de gestantes de acordo com idade e estado civil.

\begin{tabular}{lcc}
\hline Idade & $\mathbf{N}$ & $\%$ \\
\hline $14-17$ & 07 & 15,6 \\
$19-25$ & 19 & 42,2 \\
$26-29$ & 11 & 24,4 \\
$31-37$ & 08 & 17,8 \\
Estado Civil & $\mathrm{N}$ & $\%$ \\
Casada & 17 & 38,6 \\
Estável & 08 & 18,2 \\
Solteira & 18 & 40,9 \\
Outro & 01 & 2,3 \\
\hline
\end{tabular}

Fonte: próprio autor.

$\mathrm{Na}$ Tabela 2, são apresentados dados referentes às características obstétricas das gestantes. Em sua maioria, eram multigestas $(57,8 \%)$ e $86,7 \%$ das gestantes nunca abortaram.

Tabela 2 - Características obstétricas das gestantes.

\begin{tabular}{lc}
\hline Gestações Anteriores & $\%$ \\
\hline Nenhuma & 42,2 \\
Uma ou mais & 57,8 \\
\hline
\end{tabular}




\begin{tabular}{|c|c|}
\hline Gestações Anteriores & $\%$ \\
\hline Aborto & $\%$ \\
\hline 0 & 86,7 \\
\hline 1 & 8,9 \\
\hline 2 & 4,4 \\
\hline Número de partos & $\%$ \\
\hline 0 & 48,9 \\
\hline 1 & 37,8 \\
\hline 2 & 4,4 \\
\hline 3 & 8,9 \\
\hline Recém-Nascido menor de $2,5 \mathrm{~kg}$ & $\%$ \\
\hline $\operatorname{Sim}$ & 13,6 \\
\hline Não & 86,4 \\
\hline Idade Gestacional à entrevista & $\%$ \\
\hline Primeiro Trimestre & 27,0 \\
\hline Segundo Trimestre & 35,1 \\
\hline Terceiro Trimestre & 37,8 \\
\hline Consultas de Pré-Natal & $\%$ \\
\hline$<3$ consultas & 38,1 \\
\hline 3 consultas & 11,9 \\
\hline$>3$ consultas & 50,0 \\
\hline
\end{tabular}

Fonte: próprio autor.

Mais de $90 \%$ das gestantes residiam em domicílios com até cinco moradores e a maioria $(62,2 \%)$ pertencia a famílias com renda mensal de até um salário mínimo. $\mathrm{O}$ tipo de moradia mais citado foi o de alvenaria com reboco $(88,9 \%)$, e $82,2 \%$ citaram a presença do banheiro dentro de casa. Dentre os tipos de pisos, $48,9 \%$ informaram possuir, em sua residência, piso de cimento e outras $(48,9 \%)$ informaram possuir piso de cerâmica. Quanto à cobertura, $57,8 \%$ residiam em domicílios com telha, 13,3\% com laje, $6,7 \%$ com cobertura mista e 22,2\% com outros tipos de cobertura. Quanto ao tipo de esgotamento sanitário, $44 \%$ residiam em domicílios com fosse séptica, 24,4\% utilizavam a rede pública, 20\% relataram fossa negra, 2,2\% vala, $4,4 \%$ não tinham e $4,4 \%$ outro tipo.
A água utilizada para higiene pessoal e fins domésticos foi referida por $86,7 \%$ como de proveniência da rede pública. Sobre a água utilizada para beber, $42,2 \%$ referiram utilizar água mineral, 31,1\% não sabiam informar a proveniência da água, $8,9 \%$ utilizavam água coada e apenas 2,2\% água fervida. Quanto a destino do lixo, 93,3\% referiram utilizar a coleta pública de lixo. Mais da metade das gestantes $(62,2 \%)$ não criava nenhum animal em casa.

No presente estudo, $82,2 \%$ das gestantes faziam uso da suplementação de ferro e apenas 7\% foram classificadas como anêmicas.

A prevalência de parasitoses encontrada foi de $48 \%$ e, dentre estas gestantes, 66,66 \% monoparasitadas e 33,34 $\%$ poliparasitadas. Na Figura 1, observa-se que as espécies de parasitos e enterocomensais mais prevalentes foram Entamoeba coli (20\%) e E. hystolitica/E. dispar (20\%), seguidas de Iodamoeba butschlii (12\%), Giardia lamblia e Endolimax nana, com $(8 \%)$ cada.

Em relação ao estado nutricional, observou- se que $48,5 \%$ das gestantes foram classificadas na condição eutrófica, $22,9 \%$ tinham baixo peso, $17,1 \%$ sobrepeso e $11,4 \%$ estavam com obesidade.

$\mathrm{Na}$ Tabela 3, analisou-se a associação entre as variáveis socioeconômicas, como escolaridade, renda familiar e esgotamento sanitário, e a presença de parasitose nas gestantes, não sendo observada associação entre as variáveis.

$\mathrm{Na}$ análise entre o 'tipo de esgotamento sanitário' e 'positividade para o exame parasitológico' na população estudada, observou-se que $71,4 \%$ das gestantes com residências com esgotamento sanitário precário (fossa negra, vala, ou que não possuíam nenhum tipo de esgotamento sanitário) apresentaram positividade, enquanto apenas $38,9 \%$ das gestantes com residências com esgotamento sanitário adequado (rede pública coletora de esgoto e fossa séptica) apresentaram positividade. Apesar da alta prevalência de parasitoses nas gestantes residentes em locais com esgotamento sanitário precário, não houve associação estatisticamente significativa entre essas variáveis ( $p$ $=0,144)$.

Foi observado que $66,7 \%$ das gestantes com baixa escolaridade apresentaram positividade para parasitoses intestinais, e constatou-se uma prevalência de 42,1\% entre aquelas com alta escolaridade. Porém não houve associação significativa entre essas duas variáveis $(\mathrm{p}=0,294)$.

Em relação ao nível econômico, a maioria das gestantes, $62,2 \%$, possuía renda familiar de até um salário mínimo e, dentre estas, $58,8 \%$ tiveram seus exames positivos para algum parasito intestinal.

Apenas $60 \%$ das gestantes utilizavam água adequada para consumo (água mineral, água fervida ou água filtrada) e foi encontrada associação entre a procedência da água 
de consumo e a positividade para o exame parasitológico $(\mathrm{p}=0,027)$ (Tabela 3).

Figura 1 - Gráfico mostrando a distribuição dos enteroparasitas e enterocomensais em gestantes.

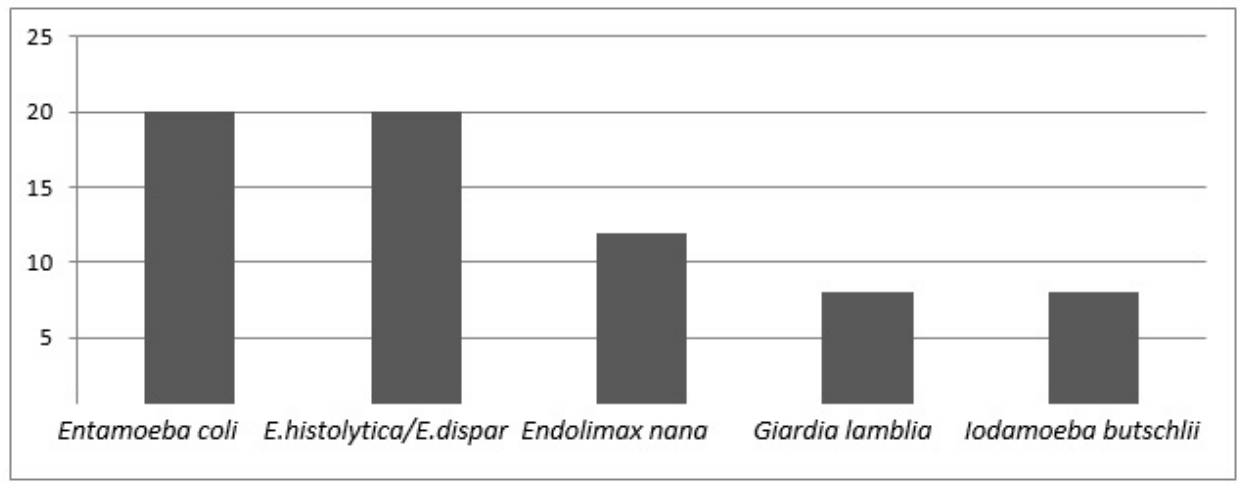

Fonte: próprio autor.

Tabela 3 - Associação entre variáveis socioeconômicas e presença de parasitoses intestinais.

\begin{tabular}{lccc}
\hline Variável & $\begin{array}{c}\text { Presença de parasitoses intestinais } \\
\text { Nim }\end{array}$ & Valor de $\mathbf{p}$ \\
\hline Escolaridade* & & & \\
Baixa escolaridade & $66,7 \%$ & $33,3 \%$ & 0,294 \\
Alta escolaridade & $42,1 \%$ & $57,9 \%$ & 0,114 \\
Renda Familiar & & & \\
Até 1 salário & $58,8 \%$ & $41,2 \%$ & 0,144 \\
Mais de 1 salário & $25 \%$ & $75 \%$ & \\
Esgotamento Sanitário** & & $61,1 \%$ & \\
Adequado & $38,9 \%$ & $28,6 \%$ & 0,027 \\
Condição de Risco & $71,4 \%$ & & \\
Procedência da Água*** & & $30,8 \%$ & \\
Adequada & $25,0 \%$ & $69,2 \%$ & \\
Inadequada & & & \\
\hline
\end{tabular}

* Consideraram-se como de Baixa Escolaridade as gestantes com Nenhum Estudo, Alfabetizadas e com Ensino Fundamental. Como de Alta Escolaridade, consideraramse as gestantes com Ensino Médio e Ensino Superior.

** Consideraram-se como tendo Esgotamento Sanitário Adequado as gestantes com residências em que o esgotamento sanitário fosse Rede Pública ou Fosse Séptica. Em Condição de Risco, consideraram-se as gestantes em cujas residências o esgotamento sanitário fosse Fossa Negra, Vala ou houvesse Ausência de Esgotamento Sanitário.

*** Considerou-se como Adequada: água fervida, água mineral ou água filtrada e Inadequada: água coada e água de procedência desconhecida.

\section{Fonte: próprio autor.}


A Tabela 4 mostra o conhecimento das gestantes em relação a parasitoses intestinais e hábitos de higiene, entre as parasitadas e as não parasitadas.

Tabela 4 - Conhecimentos sobre parasitoses intestinais e hábitos de higiene em gestantes parasitadas e não parasitadas.

\begin{tabular}{lcc}
\hline Conhecimentos sobre parasitoses intestinais & Sim & Não \\
\hline Sabe como ocorre contaminação por parasitas intestinais & $46,7 \%$ & $53,3 \%$ \\
Conhece os sintomas de uma parasitose intestinal & $28,9 \%$ & $71,1 \%$ \\
Hábitos de higiene & & $2,2 \%$ \\
Costume de lavar as mãos após utilizar o banheiro & $97,8 \%$ & $73,3 \%$ \\
Hábito de andar descalço & $26,7 \%$ & \\
\hline
\end{tabular}

Fonte: próprio autor.

\section{DISCUSSÃO}

O presente estudo mostrou que quase a metade das gestantes apresentou enteroparasitos e/ou enterocomensais na análise parasitológica das fezes. A avaliação do perfil socioeconômico demonstrou maior prevalência de gestantes com baixa renda e com pouco conhecimento sobre parasitoses intestinais. Os resultados apontam para a existência de uma população exposta a riscos de infecção por enteroparasitos, em virtude da utilização de água de procedência inadequada para consumo. A classificação do estado nutricional mostrou o predomínio da normalidade. Apesar de a anemia ser um dos estados patogênicos mais comuns em gestantes, observaram-se frequências baixas de anemia.

A escassez de pesquisas relacionadas a parasitoses intestinais e gestação dificulta comparações. No entanto, os resultados de prevalência encontrados no presente estudo foram semelhantes aos encontrados em gestantes que realizaram o acompanhamento pré-natal pelo Sistema Único de Saúde, na cidade de Viçosa - MG ${ }^{20}{ }^{20}$ Em ambos os estudos, a maior prevalência foi de protozoários patogênicos, como E. histolytica/E. díspar, e comensais intestinais, como E. coli e Endolimax nana. ${ }^{20}$ Estudo realizado com uma amostra de 316 gestantes que iniciaram o pré-natal de baixo risco do ambulatório de pré-natal do Instituto Materno Infantil de Pernambuco (IMIP) observou uma frequência de parasitoses intestinais de $37 \%,{ }^{11}$ valor inferior ao encontrado no presente estudo. Também se observou E. bistolytica/E. dispar como o parasita mais encontrado. ${ }^{11}$

É importante salientar que, apesar de elevada, a preva- lência de parasitismo encontrada pode estar subestimada, seja pelo número de amostras fecais analisadas, uma única amostra de cada gestante, ou pelo método utilizado, visto que a sedimentação espontânea não é a metodologia adequada para o diagnóstico de alguns parasitos intestinais como Strongyloides stercoralis e Enterobius vermicularis, ${ }^{19}$ sendo, então, limitações do estudo.

Apesar de os protozoários Endolimax nana e Entamoeba coli serem considerados não patogênicos, é importante destacar sua presença, pois são marcadores de contaminação fecal da gestante. ${ }^{6}$ Vale destacar que todos os protozoários encontrados apresentam em comum o mesmo mecanismo de transmissão, a ingestão de água ou alimentos contaminados por cistos ou rota fecal oral dire$\mathrm{ta}^{19}$ e chama a atenção também a ausência do encontro de helmintos entre as gestantes parasitadas. Tal situação igualmente foi evidenciada em alguns estudos realizados na região nordeste, que revelaram prevalências baixas ou nulas para o encontro de helmintos como A. lumbricoides e T. trichiura. ${ }^{21,22,23}$

O percentual das gestantes que faziam uso de ácido fólico $(80 \%)$ foi superior ao encontrado em outros estudos realizados no Brasil, em que a prevalência de suplementação com ácido fólico não chega a 40\%. ${ }^{24,25}$ Apesar de as gestantes serem um grupo vulnerável à anemia, em decorrência da elevada demanda de ferro para suprir tanto a necessidade da mãe quanto a do feto, apenas três gestantes foram classificadas como anêmicas, de acordo com diretrizes do Ministério da Saúde. ${ }^{8}$

A prevalência de anemia nas gestantes (7\%) apresentase como baixa, quando comparada aos resultados encon- 
trados em grande parte dos estudos de base populacional realizados no Brasil. Estudo com 150 gestantes na região semiárida de Alagoas encontrou 50\% de gestantes anêmicas. ${ }^{26}$ Em Viçosa - MG, verificou-se prevalência de anemia de 34,2\% ${ }^{27}$ Em outro estudo também em Viçosa - MG, encontrou-se prevalência de anemia de $28,8 \%$ nas gestantes..$^{20}$ Em Cuiabá - MT, observaram-se prevalências de anemia de $25,5 \%$ em 954 gestantes. ${ }^{28}$ Por outro lado, outros estudos identificaram prevalências diferentes, quando comparados com os estudos mencionados acima. Em Campo Mourão - PR, constatou-se uma prevalência de anemia de $6,18 \%,{ }^{29}$ inferior à encontrada em Maringá $\mathrm{PR},{ }^{28} \mathrm{em}$ que se verificou uma prevalência de 10,6\%. Ambos os estudos apresentaram prevalências de anemia próximas às verificadas neste estudo.

Já está bem estabelecida na literatura a relação do estado nutricional da gestante com fatores como risco de desenvolvimento de diabetes, hipertensão e baixo peso ao nascer. ${ }^{30,31} \mathrm{Um}$ estudo realizado com gestantes em vulnerabilidade social em Viçosa encontrou correlação das variáveis antropométricas com o peso da criança ao nascer, ${ }^{32}$ indicando que o cuidado com o estado nutricional gestacional é extremamente importante para a saúde da genitora e infantil. A classificação do estado nutricional das gestantes no presente estudo mostrou o predomínio da normalidade.

No presente estudo, variáveis socioeconômicas como escolaridade, renda familiar e esgotamento sanitário não foram associadas à presença de parasitoses nas gestantes. Diferente do estudo de Souza et al., ${ }^{11}$ que observou que a escolaridade expressa em anos de estudo esteve relacionada estatisticamente, de forma inversa, com a presença de parasitos $(\mathrm{p}=0,002)$.

Embora tenha sido encontrado um maior percentual de gestantes com baixa escolaridade positivo para parasitoses intestinais, não houve associação significativa entre essas duas variáveis $(p=0,294)$. Assim também foi demonstrado por Visser et al. ${ }^{4}$, em um inquérito de prevalência de parasitoses intestinais associado a uma avaliação das condições socioeconômicas em moradores da área periférica da cidade de Manaus - AM.

Ao estudar a ocorrência de geo-helmintíases em crianças residentes em dez municípios da região Norte-Nordeste, Fonseca et al. ${ }^{3}$ relacionam a presença de enteroparasitoses à menor renda familiar; no entanto, no presente estudo, não foi encontrada uma associação significativa ( $p=0,114)$ entre essas duas variáveis, situação também verificada por Oliveira e $\operatorname{Amor}^{23}$ em Araci, nordeste baiano.

Este estudo apresentou duas limitações - a adesão das gestantes ao exame parasitológico e a dificuldade de obtenção de dados hematológicos dos cartões das ges- tantes pela falta de registros. No entanto, a realização e a continuidade de estudos epidemiológicos são de extrema relevância, pois, com os resultados obtidos, é possível conhecer a distribuição e a frequência de casos, além de se fornecerem informações que são suporte para medidas profiláticas aos agravos presentes.

\section{CONCLUSÃO}

A prevalência de parasitoses intestinais nas gestantes pesquisadas foi de $48 \%$ e foram encontradas cinco espécies de protozoários intestinais, sendo Entamoeba coli e Entamoeba histolytica/E. dispar as mais prevalentes. Não foram observadas associações significativas entre parasitoses intestinais e as variáveis analisadas, com exceção da procedência da água utilizada para consumo. $\mathrm{O}$ estudo mostrou prevalência de anemia baixa e, embora a condição nutricional eutrófica tenha sido a mais prevalente, ainda assim preocupa a grande quantidade de gestantes acima do peso e obesas, pelo risco de comorbidades na gestação. O desconhecimento de parte das gestantes sobre como ocorre a contaminação por parasitos intestinais é um dado preocupante, pois possivelmente contribuiu para o aparecimento de enteroparasitoses. Destacam-se, então, como medidas de profilaxia primária nas gestantes, a necessidade da aplicação do diagnóstico coproparasitológico durante o prénatal, muitas vezes negligenciado, e a educação sanitária.

\section{AGRADECIMENTOS}

Ao PET Nutrição (Núcleo PENSO), pelas contribuições no tratamento estatístico. O presente trabalho foi realizado com apoio do Programa PIBIC/CNPq/UFCG Brasil.

\section{REFERÊNCIAS}

1. Scott ME. Ascaris lumbricoides: a review of its epidemiology and relationship to other infections. Ann Nestlé. 2008; 8:7-22.

2. Brum JWA, Conceição AS, Gonçalves FVC. Parasitoses oportunistas em pacientes com o vírus da imunodeficiência humana. Rev Bras Clín Med. 2013; 11(3):280-288.

3. Fonseca EOL, Teixeira MG, Barreto ML, Carmo EH, Costa MCN. Prevalência e fatores associados às geohelmintíases em crianças residentes em municípios com baixo IDH no Norte e Nordeste brasileiros. Cad Saúde Pública. 2010; 26(1):143-152. 
4. Visser S, Giatti LL, Carvalho RAC. Estudo da associação entre fatores socioambientais e prevalência de parasitose intestinal em área periférica da cidade de Manaus (AM, Brasil). Ciênc Saúde Coletiva. 2011; 16(8):3481-3492.

5. Rodriguez-Garcia R, Rodríguez-Guzmán LM, Sánchez-Maldonado MI, Gómez-Delgado A, Rivera-Cedillo R. Prevalence and risk factors associated with intestinal parasitoses in pregnant women and their relation to the infant's birth weight. Ginecol Obstet Mex. 2002; 70:338-343.

6. Santos AS, Merlini LS. Prevalência de enteroparasitoses na população do município de Maria Helena, Paraná. Ciênc Saúde Coletiva. 2010; 15(3):899-905.

7. Coura JR. Dinâmica das doenças infecciosas e parasitárias. Rio de Janeiro: Guanabara Koogan; 2008.

8. Ministério da Saúde (Brasil). Série A. Normas e Manuais Técnicos - Série Direitos Sexuais e Direitos Reprodutivos - Pré-natal e Puerpério: atenção qualificada e humanizada. Brasília: Ministério da Saúde; 2005.

9. Roucourt S, Santos ADS, Chieffi PP, Faria Junior D, Balieiro FO. Diagnóstico e tratamento das parasitoses intestinais na gestação. GO Atual. 1999; 9:23-9.

10. Weigel MM, Calle A, Armijos RX, Vega IP, Bayas BV, Montenegro CE. The effect of chronic intestinal parasitic infection on maternal and perinatal outcome. Int J Gynaecol Obstet. 1996; 52(1):9-17.

11. Souza AI, Ferreira LOC, Batista FM, Dias MRFS. Intestinal parasites, anemia and nutritional status in pregnant women in a public health care unit. Rev Bras Ginecol Obstet. 2002; 24(4):253-259.

12. Costa-Macedo LM, Rey L. Enteroparasitoses em gestantes e puérperas no Rio de Janeiro. Cad Saúde Pública. 1996; 12(3):383-388.

13. Haider BA, Humayun Q, Bhutta ZA. Effect of administration of antihelminthics for soil transmitted helminths during pregnancy. Cochrane Database Syst Rev. 2009; 15(2):CD005547.

14. Rodríguez-Morales AJ, Barbella RA, Case C, Arria M, Ravelo M, Perez H, et al. Intestinal parasitic infections among pregnant women in Venezuela. Infect Dis Obstet
Gynecol. 2006; 2006:23125.

15. Abrams B, Altman SL, Pickett KE. Pregnancy weight gain: still controversial. Am J Clin Nut. 2000; 71(5 Suppl):1233S-41S.

16. Menezes AMB, Victoria CG, Barros FC. Mortalidade infantil em duas coortes de base populacional no Sul do Brasil: tendências e diferenciais. Cad Saúde Pública. 1996; 12(1):79-86.

17. Brasil. Instituto Brasileiro de Geografia e Estatística. Cidadesat [Internet] 2010 [acesso em 2010 fev. 18]. Disponível em: http://cidades.ibge.gov.br/xtras/perfil. php?lang $=\& \operatorname{codmun}=250510$.

18. Luna-Filho B. Sequência básica na elaboração de protocolos de pesquisa. Arq Bras Cardiol. 1998; 71(6):735740 .

19. De Carli GA. Parasitologia clínica - seleção de métodos e técnicas de laboratório para o diagnóstico das parasitoses humanas. São Paulo: Atheneu; 2008.

20. Einloft ABN, Vítor CFH, Sant'Ana LFR, Priore SE, Franceschini SCC. Efeito das infecções parasitárias e da anemia materna sobre o peso ao nascer de crianças no município de Viçosa, MG. Rev Med Minas Gerais. 2010; 20(3):317-322.

21. Alves JR, Macedo HW, Ramos Júnior NA, Ferreira LF, Gonçalves MLC, Araújo A. Parasitoses intestinais em região semiárida do Nordeste do Brasil: resultados preliminares distintos das prevalências esperadas. Cad Saúde Pública. 2003; 19(2):667-670.

22.Miranda GC, Vitor CCD, Artur DL. Enteroparasitos e condições socioeconômicas e sanitárias de uma comunidade Quilombola do semiárido baiano. Rev Patol Tropical. 2010; 3(1):48-55.

23. Oliveira VF; Amor ALM. Associação entre a ocorrência de parasitos intestinais e diferentes variáveis clínicas e epidemiológicas em moradores da comunidade Ribeira I, Araci, Bahia, Brasil. Rev Bras Anal Clín. 2012; 44(1):15-25.

24. Mezzomo CLS, Garcias GL, Sclowitz ML, Sclowitz IT, Brum CB, Fontana T, et al. Prevenção de defeitos do tubo neural: prevalência do uso da suplementação de ácido fólico e fatores associados em gestantes na cidade de 
Pelotas, Rio Grande do Sul, Brasil. Cad Saúde Pública. 2007; 23(11):2716-2726.

25. Fonseca VM, Sichieri R, Brasílio L, Ribeiro LVC. Consumo de folato em gestantes de um hospital público do Rio de Janeiro. Rev Bras Epidemiol. 2003; 6(4):319-327.

26. Ferreira HS, Moura FA, Cabral JCR. Prevalência e fatores associados à anemia em gestantes da região semiárida do Estado de Alagoas. Rev Bras Ginecol Obstet. 2008; 30(9):445-451.

27. Vieira MAG. Enteroparasitoses e anemia ferropriva em gestantes assistidas na unidade saúde da família de Nova Viçosa e Posses, no município de Viçosa - MG [dissertação]. Viçosa: Universidade Federal de Viçosa; 2008.

28. Fujimori E, Sato APS, Araújo CRMA, Uchimura TTT, Porto ES, Brunken GS, et al. Anemia em gestantes de municípios das regiões Sul e Centro-Oeste do Brasil. Rev Esc Enferm USP. 2009; 43(spe2):1204-1209.

29. Américo SCM, Ferraz FN. Prevalência de anemias em gestantes do município de Campo Mourão - PR entre os períodos de 2005 a 2008. Semina: Ciênc Biol Saúde. 2011; 32(1):59-68.

30. Accioly E, Saunders C, Lacerda EMA. Nutrição em obstetrícia e pediatria. Rio de Janeiro: Guanabara Koogan; 2009.

31. Vitolo MR. Nutrição - da gestação ao envelhecimento. Rio de Janeiro: Rubia; 2008.

32. Rocha DS, Netto MP, Priore SE, Lima NMM, Rosado LEFPL, Franceschini SCC. Estado nutricional e anemia ferropriva em gestantes: relação com o peso da criança ao nascer. Rev Nutr. 2005; 18(4):481-489.

Submissão: maio de 2016

Aprovação: outubro de 2017 